\title{
APPROXIMATE DOUBLE COMMUTANTS IN VON NEUMANN ALGEBRAS AND C*-ALGEBRAS
}

\author{
DON HADWIN
}

Abstract. Richard Kadison showed that not every commutative von Neumann subalgebra of a factor von Neumann algebra is equal to its relative double commutant. We prove that every commutative $\mathrm{C}^{*}$-subalgebra of a centrally prime $\mathrm{C}^{*}$-algebra $\mathscr{B}$ equals its relative approximate double commutant. If $\mathscr{B}$ is a von Neumann algebra, there is a related distance formula.

Mathematics subject classification (2010): Primary 46L19; Secondary 46L05.

Keywords and phrases: Double commutant, approximate double commutant, hyperreflexive.

\section{REFERENCES}

[1] Marie ChODA, A condition to construct a full $I I_{1}$-factor with an application to approximate normalcy, Math. Japon. 28 (1983) 383-398.

[2] J. Dixmier, Sur les $C^{*}$-algèbres, Bull. Soc. Math. France 88 (1960) 95-112.

[3] Don Hadwin, An asymptotic double commutant theorem for $C^{*}$-algebras, Trans. Amer. Math. Soc. 244 (1978) 273-297.

[4] Don Hadwin, Approximately hyperreflexive algebras, J. Operator Theory 28 (1992) 51-64.

[5] Don Hadwin, Continuity modulo sets of measure zero, Math. Balkanica (N.S.) 3 (1989) 430-433.

[6] Don Hadwin, Vern I. PAUlsen, Two reformulations of Kadison's similarity problem, J. Operator Theory 55 (2006) 3-16.

[7] Paul Joliss aint, Operator algebras related to Thompson's group F., J. Aust. Math. Soc. 79 (2005) 231-241.

[8] RichaRd V. Kadison, Normalcy in operator algebras, Duke Math. J. 29 (1962) 459-464.

[9] RichaRd V. KADISON AND J. R. RingRose, Fundamentals of the theory of operator algebras, Vol. II, New York: Harcourt, 1986.

[10] G. G. KASPAROV, The operator K-functor and extensions of $C^{*}$-algebras, Math. USSR-Isv. 16 (1981) 513-572.

[11] Silvio Machado, On Bishop's generalization of the Weierstrass-Stone theorem, Indag. Math. 39 (1977) 218-224.

[12] T. J. RANSFORD, A short elementary proof of the Bishop-Stone-Weierstrass theorem, Math. Proc. Cambridge Philos. Soc. 96 (1984), no. 2, 309-311.

[13] Shlomo Rosenoer, Distance estimates for von Neumann algebras, Proc. Amer. Math. Soc. 86 (1982) 248-252.

[14] B. J. Vowden, Normalcy in von Neumann algebras, Proc. London Math. Soc. (3) 27 (1973) 88-100.

[15] Nik Weaver, A prime $C^{*}$-algebra that is not primitive, J. Funct. Anal. 203 (2003) 356-361. 